\title{
Generalized Cauchy identities, trees and multidimensional Brownian motions. Part I: bijective proof of generalized Cauchy identities
}

\author{
Piotr Śniady \\ Institute of Mathematics, \\ University of Wroclaw, \\ pl. Grunwaldzki 2/4, \\ 50-384 Wroclaw, Poland \\ Piotr.Sniady@math. uni.wroc.pl
}

Submitted: Jul 3, 2006; Accepted: Jul 17, 2006; Published: Aug 3, 2006

Mathematics Subject Classification: 60J65; 05A19

\begin{abstract}
In this series of articles we study connections between combinatorics of multidimensional generalizations of the Cauchy identity and continuous objects such as multidimensional Brownian motions and Brownian bridges.

In Part I of the series we present a bijective proof of the multidimensional generalizations of the Cauchy identity. Our bijection uses oriented planar trees equipped with some linear orders.
\end{abstract}

\section{Introduction}

Since this paper constitutes the Part I of a series of articles we allow ourself to start with a longer introduction to the whole series.

\subsection{Toy example}

The goal of this series of articles is to discuss multidimensional analogues of the Cauchy identity. However, before we do this and study our problem in its full generality, we would like to have a brief look on the simplest case of the (usual) Cauchy identity. Even in this simplified setting we will be able to see some important features of the general case. 


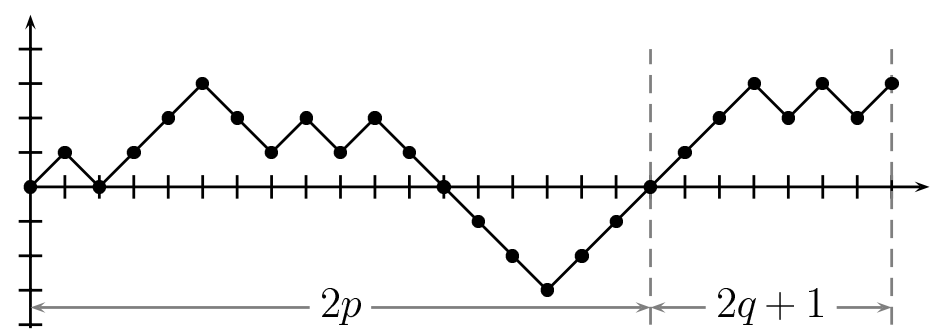

Figure 1: A graphical representation of the sequence $\left(x_{i}\right)_{1 \leq i \leq 25}=(1,-1,1,1,1,-1, \ldots)$. It is also a graph of a continuous piecewise affine function $X:[0,25] \rightarrow \mathbb{R}$ which is canonically associated to the sequence $\left(x_{i}\right)$.

\subsubsection{Cauchy identity}

Cauchy identity states that for each nonnegative integer $l$

$$
2^{2 l}=\sum_{p+q=l}\left(\begin{array}{c}
2 p \\
p
\end{array}\right)\left(\begin{array}{c}
2 q \\
q
\end{array}\right)
$$

where the sum runs over nonnegative integers $p, q$. In order to give a combinatorial meaning to this identity we interpret the left-hand side of (1) as the number of sequences $\left(x_{1}, \ldots, x_{2 l+1}\right)$ such that $x_{1}, \ldots, x_{2 l+1} \in\{-1,1\}$ and $x_{1}+\cdots+x_{2 l+1}>0$. For each such a sequence $\left(x_{i}\right)$ we set $p \geq 0$ to be the biggest integer such that $x_{1}+\cdots+x_{2 p}=0$ and set $q=l-p$; it follows that $\left(x_{i}\right)$ is a concatenation of sequences $\left(y_{1}, \ldots, y_{2 p}\right)$ and $\left(z_{0}, z_{1}, \ldots, z_{2 q}\right)$, where $y_{1}+\cdots+y_{2 p}=0$ and all partial sums of the sequence $\left(z_{i}\right)$ are positive: $z_{0}+\cdots+z_{i}>0$ for all $1 \leq i \leq 2 q$. This can be illustrated graphically as follows: we treat the sequence $\left(x_{i}\right)$ as a random walk and $2 p$ is the time of the last return of the trajectory to its origin, of Figure 1 Clearly, for each value of $p$ there are $\left(\begin{array}{c}2 p \\ p\end{array}\right)$ ways of choosing the sequence $\left(y_{i}\right)$ and it is much less obvious (we shall discuss this problem later

on) that for each value of $q$ there are exactly $\left(\begin{array}{c}2 q \\ q\end{array}\right)$ ways of choosing the sequence $\left(z_{i}\right)$; in this way we found a combinatorial interpretation of the right-hand side of the Cauchy identity (11).

\subsubsection{Bijective proof and Pitman transform}

In the above discussion we used without a proof the fact that the number of the sequences $\left(z_{0}, \ldots, z_{2 q}\right)$ is equal to $\left(\begin{array}{c}2 q \\ q\end{array}\right)$. The latter number has a clear combinatorial interpretation as the number of sequences $\left(t_{1}, \ldots, t_{2 q}\right)$ with $t_{1}, \ldots, t_{2 q} \in\{-1,1\}$ and $t_{1}+\cdots+t_{2 q}=0$, it would be therefore very tempting to proof the above statement by constructing a bijection between the sequences $\left(z_{i}\right)$ and the sequences $\left(t_{i}\right)$ and we shall do it in the following.

Firstly, instead of considering the sequences $\left(z_{0}, \ldots, z_{2 q}\right)$ of length $2 q+1$ with all partial sums positive it will be more convenient to skip the first element and to consider sequences $\left(z_{1}, \ldots, z_{2 q}\right)$ of length $2 q$ such that $z_{1}, \ldots, z_{2 q} \in\{-1,1\}$ with all partial sums nonnegative: $z_{1}+\cdots+z_{i} \geq 0$ for all $1 \leq i \leq 2 q$. 
Secondly, it will be convenient to represent the sequences $\left(z_{1}, \ldots, z_{2 q}\right)$ and $\left(t_{1}, \ldots, t_{2 q}\right)$ as continuous piecewise affine functions $Z, T:[0,2 q] \rightarrow \mathbb{R}$ just as we did on Figure 1. Formally, function $Z$ is defined as the unique continuous function such that $Z(0)=0$ and such that for each integer $1 \leq i \leq 2 q$ we have $Z^{\prime}(s)=z_{i}$ for all $s \in(i-1, i)$. In this way we can assign a function to any sequence consisting of only 1 and -1 and we shall make use of this idea later on.

It turns out that an example of a bijection between sequences $\left(t_{i}\right)$ and $\left(z_{i}\right)$ is provided by the Pitman transform [Pit75] which to a function $T$ associates a function

$$
Z_{s}=T_{s}-2 \inf _{0 \leq r \leq s} T_{r}
$$

We shall analyze this bijection in a more general context in Part III [JŚ06b] of this series.

\subsubsection{Brownian motion limit and arc-sine law}

What happens to the combinatorial interpretation of the Cauchy identity (1) when $l$ tends to infinity? We define a rescaled function $\tilde{X}_{s}:[0,1] \rightarrow \mathbb{R}$ given by

$$
\tilde{X}_{s}=\frac{1}{\sqrt{2 l+1}} X_{(2 l+1) s},
$$

where $X:[0,2 l+1] \rightarrow \mathbb{R}$ is the usual function associated to the sequence $x_{1}, \ldots, x_{2 l+1}$ as on Figure 1. The normalization factors were chosen in such a way that if the sequence $\left(x_{i}\right)$ is taken randomly (provided $x_{1}+\cdots+x_{2 l+1}>0$ ) then the stochastic processes $\tilde{X}_{s}$ converge in distribution (as $l$ tends to infinity) to the Brownian motion $B:[0,1] \rightarrow \mathbb{R}$ conditioned by a requirement that $B_{1} \geq 0$.

It follows that random variables $\tilde{\Theta}=\sup \left\{t \in[0,1]: \tilde{X}_{t}=0\right\}$ converge in distribution (as $l$ tends to infinity) to a random variable $\Theta=\sup \left\{t \in[0,1]: B_{t}=0\right\}$, the time of the last visit of the trajectory of the Brownian motion in the origin. The discussion from Sections 1.1 .1 and 1.1 .2 shows that the distribution of the random variable $\tilde{\Theta}$ is given explicitly by

$$
P(\tilde{\Theta}<x)=\sum_{\substack{p+q=l \\
p<x(2 l+1)}} \frac{\left(\begin{array}{c}
2 p \\
p
\end{array}\right)\left(\begin{array}{c}
2 q \\
q
\end{array}\right)}{2^{2 l}} .
$$

The distribution of the random variable $\Theta$ does not change when we replace the Brownian motion $\left(B_{t}\right)$ conditioned to fulfill $B_{1}>0$ by the usual Brownian motion. Thus, Eq. (3) after applying the Stirling formula and simple transformations implies the following well-known result.

Theorem 1 (Arc-sine law). If $\left(B_{s}\right)$ is a Brownian motion then the distribution of the random variable $\Theta=\sup \left\{t \in[0,1]: B_{t}=0\right\}$ is given by

$$
P(\Theta<x)=\frac{1}{2}+\frac{1}{\pi} \sin ^{-1}(2 x-1)
$$

for all $0 \leq x \leq 1$. 


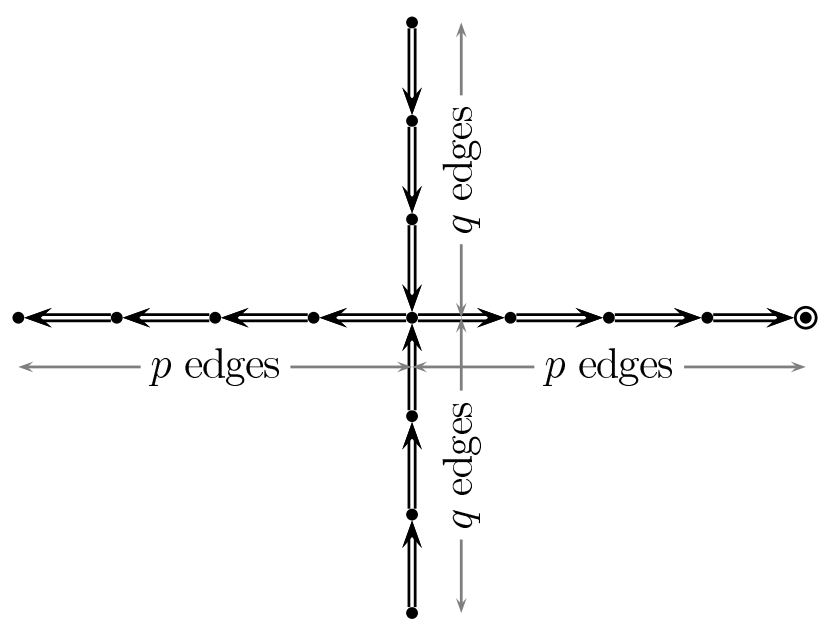

Figure 2: There are $\left(\begin{array}{c}2 p \\ p\end{array}\right)\left(\begin{array}{c}2 q \\ q\end{array}\right)$ total orders < on the vertices of this oriented tree which are compatible with the orientation of the edges.

\subsection{How to generalize the Cauchy identity?}

As we have seen above, the Cauchy identity (11) has all properties of a wonderful mathematical result: it is not obvious, it has interesting applications and it is beautiful. It is therefore very tempting to look for some more identities which would share some resemblance to the Cauchy identity or even find some general identity, equation (1) would be a special case of.

Guessing how the left-hand side of (11) could be generalized is not difficult and something like $m^{m l}$ is a reasonable candidate. Unfortunately, it is by no means clear which sum should replace the right-hand side of (1). The strategy of writing down lots of wild and complicated sums with the hope of finding the right one by accident is predestined to fail. It is much more reasonable to find some combinatorial objects which are counted by the right-hand side of (1) and then to find a reasonable generalization of these objects.

For fixed integers $p, q \geq 0$ we consider the tree from Figure 2. Every edge of this tree is oriented and it is a good idea to regard these edges as one-way-only roads: if vertices $x$ and $y$ are connected by an edge and the arrow points from $y$ to $x$ then the travel from $y$ to $x$ is permitted but the travel from $x$ to $y$ is not allowed. This orientation defines a partial order $\prec$ on the set of the vertices: we say that $x \prec y$ if it is possible to travel from the vertex $y$ to the vertex $x$ by going through a number of edges (in order to remember this convention we suggest the Reader to think that $\prec$ is a simplified arrow $\leftarrow$ ). Let $<$ be a total order on the set of the vertices. We say that $<$ is compatible with the orientations of the edges if for all pairs of vertices $x, y$ such that $x \prec y$ we also have $x<y$. It is very easy to see that for the tree from Figure 2 there are $\left(\begin{array}{c}2 p \\ p\end{array}\right)\left(\begin{array}{c}2 q \\ q\end{array}\right)$ total orders $<$ which are compatible with the orientations of the edges which coincides with the summand on the right-hand side of (1).

It remains now to find some natural way of generating the trees of the form depicted on Figure 2 with the property $p+q=l$. We shall do it in the following. 


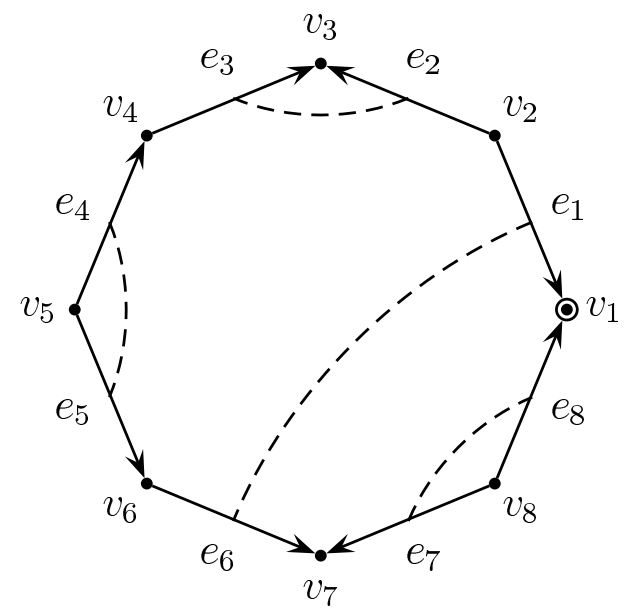

Figure 3: A graph $G_{\epsilon}$ corresponding to the sequence $\epsilon=(+1,-1,+1,+1,-1,-1,+1,-1)$. The dashed lines represent the pairing $\sigma=\{\{1,6\},\{2,3\},\{4,5\},\{7,8\}\}\}$.

\subsection{Quotient graphs and quotient trees}

We recall now the construction of Dykema and Haagerup DH04a. For integer $k \geq 1$ let $G$ be an oriented $k$-gon graph with consecutive vertices $v_{1}, \ldots, v_{k}$ and edges $e_{1}, \ldots, e_{k}$ (edge $e_{i}$ connects vertices $v_{i}$ and $v_{i+1}$ ). The vertex $v_{1}$ is distinguished, see Figure 3 . We encode the information about the orientations of the edges in a sequence $\epsilon(1), \ldots, \epsilon(k)$ where $\epsilon(i)=+1$ if the arrow points from $v_{i+1}$ to $v_{i}$ and $\epsilon(i)=-1$ if the arrow points from $v_{i}$ to $v_{i+1}$. The graph $G$ is uniquely determined by the sequence $\epsilon$ and sometimes we will explicitly state this dependence by using the notation $G_{\epsilon}$.

Let $\sigma=\left\{\left\{i_{1}, j_{1}\right\}, \ldots,\left\{i_{k / 2}, j_{k / 2}\right\}\right\}$ be a pairing of the set $\{1, \ldots, k\}$, i.e. pairs $\left\{i_{m}, j_{m}\right\}$ are disjoint and their union is equal to $\{1, \ldots, k\}$. We say that $\sigma$ is compatible with $\epsilon$ if

$$
\epsilon(i)+\epsilon(j)=0 \quad \text { for every }\{i, j\} \in \sigma .
$$

It is a good idea to think that $\sigma$ is a pairing between the edges of $G$, see Figure 3 , For each $\{i, j\} \in \sigma$ we identify (or, in other words, we glue together) the edges $e_{i}$ and $e_{j}$ in such a way that the vertex $v_{i}$ is identified with $v_{j+1}$ and vertex $v_{i+1}$ is identified with $v_{j}$ and we denote by $T_{\sigma}$ the resulting quotient graph. Since each edge of $T_{\sigma}$ origins from a pair of edges of $G$, we draw all edges of $T_{\sigma}$ as double lines. The condition (4) implies that each edge of $T_{\sigma}$ carries a natural orientation, inherited from each of the two edges of $G$ it comes from, see Figure 4 .

From the following on, we consider only the case when the quotient graph $T_{\sigma}$ is a tree. One can show [DH04a that the latter holds if and only if the pairing $\sigma$ is noncrossing [Kre72]; in other words it is not possible that for some $p<q<r<s$ we have $\{p, r\},\{q, s\} \in \sigma$. The name of the non-crossing pairings comes from their property that on their graphical depictions (such as Figure 3) the lines do not cross. Let the root $R$ of the tree $T_{\sigma}$ be the vertex corresponding to the distinguished vertex $v_{1}$ of the graph $G$. 


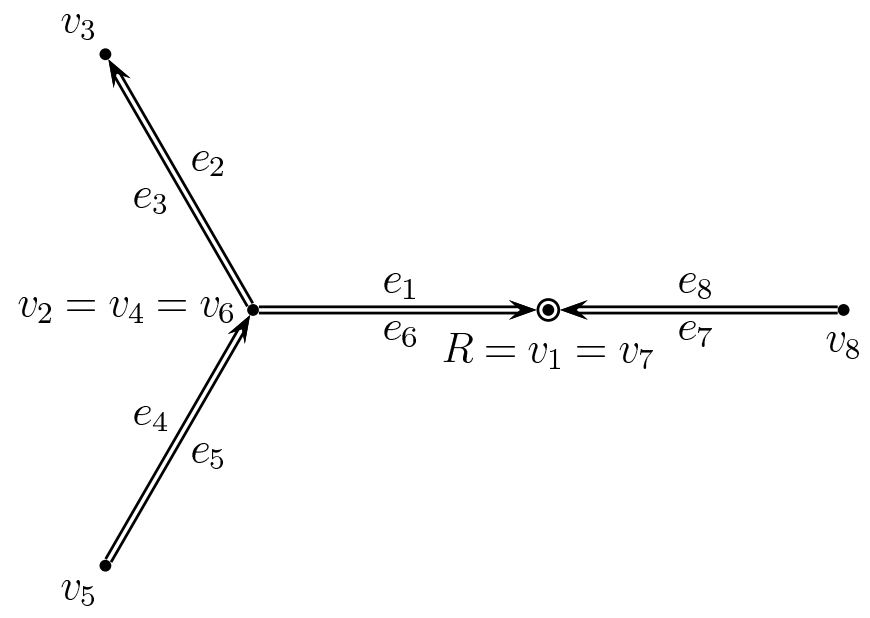

Figure 4: The quotient graph $T_{\sigma}$ corresponding to the graph from Figure 3. The root $R$ of the tree $T_{\sigma}$ is encircled.

\subsection{How to generalize the Cauchy identity? (continued)}

Let us come back to the discussion from Section 1.2. We consider the polygon $G_{\epsilon}$ corresponding to

$$
\epsilon=(\underbrace{+1}_{l \text { times }}, \underbrace{-1}_{l \text { times }}, \underbrace{+1}_{l \text { times }}, \underbrace{-1}_{l \text { times }}) .
$$

All possible non-crossing pairings $\sigma$ which are compatible with $\epsilon$ are depicted on Figure 5 and it easy to see that the corresponding quotient tree $T_{\sigma}$ has exactly the form depicted on Figure 2.

In this way we managed to find relatively natural combinatorial objects, the number of which is given by the right-hand side of the Cauchy identity (1). After some guesswork we end up with the following conjecture (please note that the usual Cauchy identity (1) corresponds to $m=2$ ).

Theorem 2 (Generalized Cauchy identity). For integers $l, m \geq 1$ there are exactly $m^{m l}$ pairs $(\sigma,<)$, where $\sigma$ is a non-crossing pairing compatible with

$$
\epsilon=(\underbrace{\underbrace{+1}_{\text {ltimes }}, \underbrace{-1}_{\text {times }}, \underbrace{+1}_{\text {l times }}, \underbrace{-1}_{\text {ltimes }}, \ldots)}_{2 m \text { blocks, i.e. total of } 2 m l \text { elements }}
$$

and $<$ is a total order on the vertices of $T_{\sigma}$ which is compatible with the orientations of the edges.

Above we provided only vague heuristical arguments why the above conjecture could be true. Surprisingly, as we shall see in the following, Theorem 2 is indeed true.

The formulation of Theorem 2 is combinatorial and therefore appears to be far from its motivation, the usual Cauchy identity (1), which is formulated algebraically, nevertheless for each fixed value of $m$ one can enumerate all 'classes' of pairings compatible with (5) 


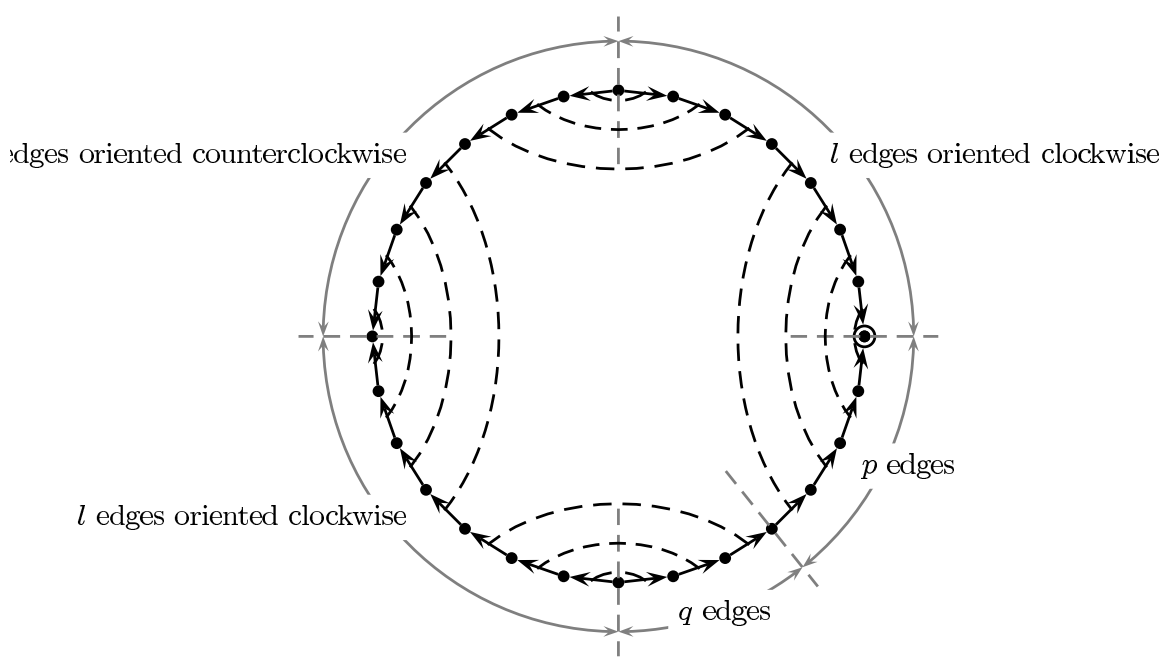

Figure 5: A graph $T$ corresponding to sequence $\epsilon=(\underbrace{+1}_{l \text { times }}, \underbrace{-1}_{l \text { times }}, \underbrace{+1}_{l \text { times }}, \underbrace{-1}_{l \text { times }})$. The dashed lines denote a pairing $\sigma$ for which the quotient graph $T_{\sigma}$ is depicted on Figure 2

and for each class count the number of compatible orders $<$. To give to the Reader a flavor of the algebraic implications of Theorem 2, we present the case of $m=3$ [DY03]

$$
3^{3 l}=\sum_{p+q=l}\left(\begin{array}{c}
3 p \\
p, p, p
\end{array}\right)\left(\begin{array}{c}
3 q \\
q, q, q
\end{array}\right)+3 \sum_{\substack{p+q+r=l-1 \\
r^{\prime}+q^{\prime}=r+q+1 \\
p^{\prime \prime}+r^{\prime \prime}=p+r+1}}\left(\begin{array}{c}
2 p+p^{\prime \prime} \\
p, p, p^{\prime \prime}
\end{array}\right)\left(\begin{array}{c}
2 q+q^{\prime} \\
q, q, q^{\prime}
\end{array}\right)\left(\begin{array}{c}
r+r^{\prime}+r^{\prime \prime} \\
r, r^{\prime}, r^{\prime \prime}
\end{array}\right) .
$$

The complication of the formula grows very quickly and already for $m=4$ the appropriate expression has a length of a half page of a typed text [Śni03.

\subsection{Historical overview: operator algebras, free probability and triangular operator $T$}

The history presented in Sections 1.21 .4 of finding the generalization of the Cauchy identity is too nice to be true and indeed it is not the way how Theorem 2 was postulated. As we shall see in the following, the path towards this result led not through combinatorics but through the theory of operator algebras. Since this section is very loosely connected with the rest of this article, Readers not interested in theory operator algebras may skip it without much harm.

\subsubsection{Invariant subspace conjecture}

One of the fundamental problems of the theory of operator algebras is the invariant subspace conjecture which asks if for every bounded operator $x$ acting on an infinitedimensional Hilbert space $\mathcal{H}$ there exists a closed subspace $\mathcal{K} \subset \mathcal{H}$ such that $\mathcal{K}$ is nontrivial 
in the sense that $\mathcal{K} \neq\{0\}, \mathcal{K} \neq \mathcal{H}$ and which is an invariant subspace of $x$. Since for many decades nobody was able to prove the invariant subspace conjecture in its full generality, Dykema and Haagerup took the opposite strategy and tried to construct explicitly a counterexample by the means of the Voiculescu's free probability theory.

The free probability [VDN92, HP00] is a non-commutative probability theory with the classical notion of independence replaced by the notion of freeness. Natural examples which fit nicely into the framework of the free probability include large random matrices, free products of von Neumann algebras and asymptotics of large Young diagrams. Families of operators which arise in the free probability are, informally speaking, very non-commutative and for this reason they are perfect candidates for counterexamples to the conjectures in the theory of operator algebras [Voi96].

The first candidate for a counterexample to the invariant subspace conjecture considered by Dykema and Haagerup was the circular operator, which unfortunately turned out to have a large family of invariant subspaces [DH01, ŚS01]. Later on Haagerup [Haa01] proved a version of a spectral theorem for certain non-normal operators and thus he constructed invariant subspaces for many classes of operators. This result gave very strong restrictions on the form of a possible counterexample, namely the Brown spectral measure [Bro86] of such an operator should be concentrated in only one point. It was a hint to look for counterexamples among, so-called, quasinilpotent operators. In this way Dykema and Haagerup [DH04a] initiated a study of the triangular operator $T$, which appeared at that time to be a perfect candidate because it is quasinilpotent and it admits very nice random matrix models.

\subsubsection{Triangular operator $T$}

The triangular operator $T$ [DH04a can be abstractly described as an element of a von Neumann algebra $\mathfrak{A}$ equipped with a finite normal faithful tracial state $\phi: \mathfrak{A} \rightarrow \mathbb{C}$ with the non-commutative moments $\phi\left(T^{\epsilon(1)} \cdots T^{\epsilon(n)}\right)$ given by

$$
\phi\left(T^{\epsilon(1)} \cdots T^{\epsilon(n)}\right)=\lim _{N \rightarrow \infty} \frac{1}{N} \mathbb{E} \operatorname{Tr}\left(T_{N}^{\epsilon(1)} \cdots T_{N}^{\epsilon(n)}\right)
$$

for any $n \in \mathbb{N}$ and $\epsilon(1), \ldots, \epsilon(n) \in\{-1,+1\}$, where we use the notation $T^{+1}:=T$ and $T^{-1}:=T^{\star} ;$ and where

$$
T_{N}=\left[\begin{array}{ccccc}
t_{1,1} & t_{1,2} & \cdots & t_{1, n-1} & t_{1, n} \\
0 & t_{2,2} & \cdots & t_{2, n-1} & t_{2, n} \\
\vdots & & \ddots & \vdots & \vdots \\
& & & t_{n-1, n-1} & t_{n-1, n} \\
0 & & \cdots & 0 & t_{n, n}
\end{array}\right]
$$

is an upper-triangular random matrix, the entries $\left(t_{i, j}\right)_{1 \leq i \leq j \leq N}$ of which are independent centered Gaussian random variables with variance $\frac{1}{N}$.

Definition (7) is not very convenient and one can show [DH04a] that it is equivalent to the following one: $(n / 2+1) ! \phi\left(T^{\epsilon(1)} \cdots T^{\epsilon(n)}\right)$ is equal to the number of pairs $(\sigma,<)$ 
such that $\sigma$ is a pairing compatible with $\epsilon$ and $<$ is a total order on the vertices of $T_{\sigma}$ which is compatible with the orientation of the edges. The Reader may easily see that the latter definition of $T$ is very closely related to the results presented in this paper; in particular Theorem 2 can be now equivalently stated as follows (in fact it is the form in which Dykema and Haagerup stated originally their conjecture [DH04a]):

Theorem 3. If $l, m \geq 1$ are integers then

$$
\phi\left[\left(T^{l}\left(T^{\star}\right)^{l}\right)^{m}\right]=\frac{m^{m l}}{(m l+1) !} .
$$

Yet another approach to $T$ is connected with the combinatorial approach to operatorvalued free probability [Spe98], namely $T$ can be described as a certain generalized circular element. Speaking very briefly, the non-commutative moments of $T$ can be described as certain iterated integrals Śni03. This approach turned out to be very fruitful: in this way in our previous work [Śni03] we found the first proof of Theorem 2 and Theorem 3, a different proof was later presented in $\mathrm{AH} 04$. Some other combinatorial results concerning the non-commutative moments of $T$ were obtained in [DY03].

Theorem 2 and Theorem 3 were conjectured by Dykema and Haagerup [DH04a] in the hope that they might be useful in the study of spectral properties of $T$. Literally speaking, this hope turned out to be wrong since the later construction of the hyperinvariant subspaces of $T$ by Dykema and Haagerup DH04b, Haa02 did not make use of Theorem 2 and Theorem 3, however it made use of one of the auxiliary results used in our proof Ś́ni03] of these theorems. In this way, indirectly, Theorem 2 and Theorem 3 turned out to be indeed helpful for their original purpose.

As we already mentioned, Dykema and Haagerup [DH04b, Haa02] constructed a family of hyperinvariant subspaces of $T$ and in this way the original motivation for studying the operator $T$ (as a possible counterexample for the invariant subspace conjecture) ended up as a failure. There are still some investigations of the triangular operator $T$ as a possible counterexample for some other conjectures, for example Aag04, however most of the specialists do not expect any surprises in the theory of operator algebras coming from this direction. In this article we would like to convince the Reader that the applications of the triangular operator $T$ in combinatorics and the classical probability theory constitute a sufficient compensation for the lost hopes concerning its applications in the theory of operator algebras.

\subsection{Overview of this series of articles}

\subsubsection{Part I: Bijective proof of generalized Cauchy identities}

In this article we shall prove the following result. Let $l_{1} \leq \cdots \leq l_{m}$ be a weakly increasing sequence of positive integers; we denote $L=l_{1}+\cdots+l_{m}+1$. For $1 \leq i \leq m$ we set

$$
\epsilon_{i}=(\underbrace{+1}_{l_{i} \text { times }}, \underbrace{-1}_{l_{i-1} \text { times }}, \ldots, \underbrace{(-1)^{i-1}}_{l_{1} \text { times }}, \underbrace{(-1)^{i}}_{l_{1} \text { times }}, \ldots, \underbrace{+1}_{l_{i-1} \text { times }}, \underbrace{-1}_{l_{i} \text { times }}),
$$


where $\underbrace{a}_{l \text { times }}$ denotes $\underbrace{a, \ldots, a}_{l \text { times }}$. The Reader may restrict his/her attention to the most interesting case when $l_{1}=l_{2}=\cdots=l$ are all equal and $\epsilon_{i}$ takes a simpler form

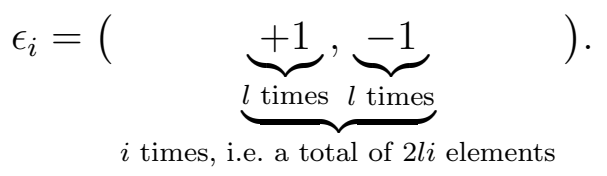

In this case $\epsilon_{m}$ coincides with (5) and it is easy to show from the Raney lemma [Ran60] that the set $(\beta)$ below has $m^{m l}$ elements [Śni03] hence Theorem 2 will follow from the following stronger result.

Theorem 4 (The main result). Let $\epsilon_{m}$ be as above. The algorithm MainBijection described in this article provides a bijection between

$(\alpha)$ the set of pairs $(\sigma,<)$, where $\sigma$ is a pairing compatible with $\epsilon_{m}$ and $<$ is a total order on the vertices of $T_{\sigma}$ which is compatible with the orientations of the edges;

$(\beta)$ the set of tuples $\left(B_{1}, \ldots, B_{m}\right)$, where $B_{1}, \ldots, B_{m}$ are disjoint sets such that $B_{1} \cup$ $\cdots \cup B_{m}=\{1, \ldots, L\}$ and

$$
\left|B_{1}\right|+\cdots+\left|B_{n}\right| \leq l_{1}+\cdots+l_{n}
$$

holds true for each $1 \leq n \leq m-1$;

Alternatively, set $(\beta)$ can be described as

$(\gamma)$ the set of sequences $\left(a_{1}, \ldots, a_{L}\right)$ such that $a_{1}, \ldots, a_{L} \in\{1, \ldots, m\}$ and for each $1 \leq n \leq m-1$ at most $l_{1}+\cdots+l_{n}$ elements of the sequence $\left(a_{i}\right)$ belong to the set $\{1, \ldots, n\}$;

where the bijection between sets $(\beta)$ and $(\gamma)$ is given by $B_{j}=\left\{k: a_{k}=j\right\}$.

Remark 5 . The sequence $\left(\tilde{a}_{1}, \ldots, \tilde{a}_{L}\right)$ can be regarded as a generalized parking function, where $\tilde{a}_{r}=m+1-a_{r}$. Indeed, let $\left(b_{1}, \ldots, b_{L}\right)$ be its non-decreasing rearrangement; then the original sequence $\left(a_{1}, \ldots, a_{L}\right)$ contributes to $(\gamma)$ iff $b_{1}, \ldots, b_{L}$ are positive integers such that $b_{1+l_{m}} \leq 1, b_{1+l_{m}+l_{m-1}} \leq 2, \ldots, b_{1+l_{m}+\cdots+l_{1}} \leq m$ which is a slighlty modified definition of a parking function.

The bijection provided by the above theorem plays the central role in this series of articles.

\subsubsection{Part II: Combinatorial differential calculus [JŚ06a]}

The bijections considered in Part I of this series (Section 3 and Section 4 of this article) are far from being trivial and the Reader might wonder how did the author guess their correct form and what is the conceptual idea behind them. To answer these questions we would like to come back to our previous work [Śni03] where we provided the first proof 
of Theorem 2. The main idea was to associate a polynomial of a single variable to every pair $(\sigma,<)$ and by additivity to every graph $G_{\epsilon}$. The polynomials associated to $\epsilon$ as in (5) with different values of $m$ turned out to be related by a simple differential equation and for this reason can be regarded as generalizations of Abel polynomials.

In Part II of this series [JŚ06a] (joint work with Artur Jeż) we present an analogue of the differential calculus in which the role of polynomials is played by certain ordered sets and trees. Our combinatorial calculus has all nice features of the usual calculus and has an advantage that the elements of the considered ordered sets might carry some additional information. In this way our analytic proof from Ś́ni03] can be directly reformulated in our new language of the combinatorial calculus; furthermore the additional information carried by the vertices determines uniquely the bijections presented in Part I of this series.

\subsubsection{Part III: Multidimensional arc-sine laws JŚ06b]}

In Section 1.1.3 we presented how a bijective proof the usual Cauchy identity can be used to extract some information about the behavior of the Brownian motion and in particular to show the arc-sine law. It is therefore natural to ask if the bijective proof of the generalized Cauchy identities presented in Part I could provide some information about multidimensional Brownian motions.

In order to answer these questions we study in Part III of this series the asymptotic behavior of the trees and bijections presented in Part I. Asymptotically, as their size tend to infinity, these trees converge towards continuous objects such as multidimensional Brownian motions and Brownian bridges. Our bijection behaves nicely in this asymptotic setting and becomes a map between certain classes of functions valued in $\mathbb{R}^{m-1}$, which is closely related to the Pitman transform and Littelmann paths. In this way we are able to describe certain interesting properties of multidimensional Brownian motions and in particular we prove a multidimensional analogue of the arc-sine law.

\section{The main bijection}

\subsection{Structure of a planar tree. Order $\triangleleft$}

For a non-crossing pairing $\sigma$ we can describe the process of creating the quotient graph as follows: we think that the edges of the graph $G$ are sticks of equal lengths with flexible connections at the vertices. Graph $G$ is lying on a flat surface in such a way that the edges do not cross. For each pair $\{i, j\} \in \sigma$ we glue together edges $e_{i}$ and $e_{j}$ by bending the joints in such a way that the sticks should not cross. In this way $T_{\sigma}$ has a structure of a planar tree, i.e. for each vertex we can order the adjacent edges up to a cyclic shift (just like points on a circle). We shall provide an alternative description of this planar structure in the following.

Let us visit the vertices of $G$ in the usual cyclic order $v_{1}, v_{2}, \ldots, v_{k}, v_{1}$ by going along the edges $e_{1}, \ldots, e_{k}$; by passing to the quotient graph $T_{\sigma}$ we obtain a journey on the graph $T_{\sigma}$ which starts and ends in the root $R$. The structure of the planar tree defined above 


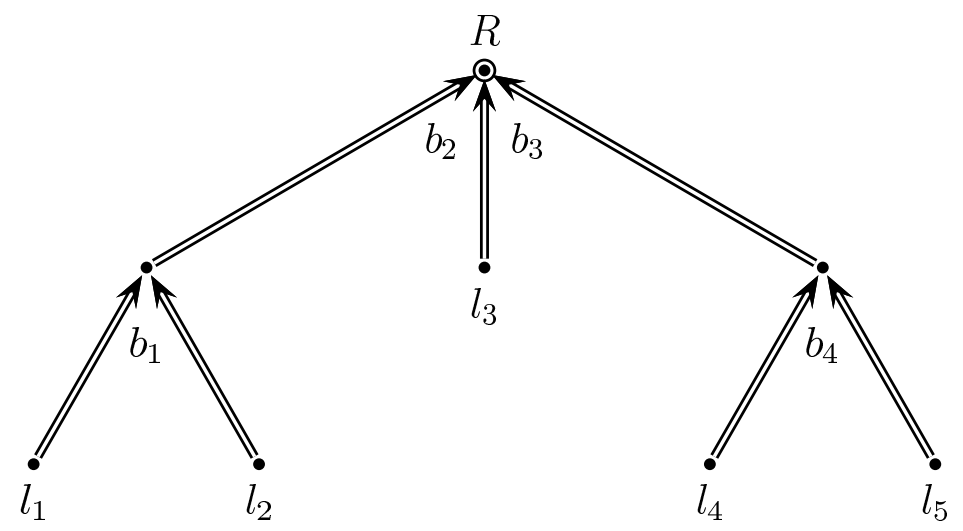

Figure 6: Example of a tree such that the arrows on all the edges point towards the root. Leafs $l_{1}, l_{2}, \ldots$ and bays $b_{1}, b_{2}, \ldots$ are marked.

can be described as follows: if we travel on the graphical representation of $T_{\sigma}$ by touching the edges by our left hand, we obtain the same journey. For each vertex of $T_{\sigma}$ we mark the time we visit it for the first time; comparison of these times gives us a total order $\triangleleft$, called preorder Sta99], on the vertices of $T_{\sigma}$. For example, in the case of the tree from Figure 4 we have $v_{1} \triangleleft v_{2} \triangleleft v_{3} \triangleleft v_{5} \triangleleft v_{8}$.

\subsection{Pairing between leafs and bays}

Suppose that $U$ is an oriented planar tree with the property that the arrows on all the edges are pointing towards the root $R$; in other words $R \preceq x$ holds true for every vertex $x$. We shall also assume that the tree $U$ consists of at least two vertices.

We call a pair of edges $\{e, f\}$ a bay if edges $e, f$ share a common vertex $v$ and are adjacent edges (adjacent with respect to the structure of the planar tree) and arrows on $e$ and $f$ point towards the common vertex $v$. It is convenient to represent a bay as the corner between edges $e$ and $f$, cf Figure 6.

A vertex is called a leaf if it is connected to exactly one edge and it is different from the root $R$, cf Figure 6 .

Let us travel on the tree $U$ (we begin and end at the root $R$ ) in such a way that we always touch the edges of the tree by our left hand. We say that a passage along an edge is negative if the arrow on the edge coincides with the direction of travel; otherwise we call it a positive passage (the origin of this convention is the following: if $U=T_{\sigma}$ is a quotient tree coming from a polygonal graph $G_{\epsilon}$, where $\epsilon=(\epsilon(1), \ldots, \epsilon(k))$ then the sign of the $n$-th step coincides with the sign of $\epsilon(n))$. It is easy to see that a bay corresponds to a pair of consecutive passages: a negative and a positive one; similarly entering and leaving a leaf corresponds to a pair of consecutive passages: a positive and a negative one. In other words, the bays and the leafs correspond to the changes in the sign of the passage. Since our journey begins with a positive passage and ends with a negative one, therefore leafs $l_{1}, \ldots, l_{p+1}$ and bays $b_{1}, \ldots, b_{p}$ are visited in the intertwining order $l_{1}, b_{1}, l_{2}, b_{2}, \ldots, l_{p}, b_{p}, l_{p+1}$. The number of the leafs (with the last leaf $l_{p+1}$ excluded) is 
equal to the number of the bays, we can therefore consider a pairing between them given by $l_{i} \mapsto b_{i}$ for $1 \leq i \leq p$. In other words, to a leaf $l$ we assign the first bay which is visited in our journey after leaving $l$.

\subsection{Catalan sequences}

We say that $\epsilon=(\epsilon(1), \ldots, \epsilon(k))$ is a Catalan sequence if $\epsilon(1), \ldots, \epsilon(k) \in\{-1,+1\}$, $\epsilon(1)+\cdots+\epsilon(k)=0$ and all partial sums are non-negative: $\epsilon(1)+\cdots+\epsilon(l) \geq 0$ for all $1 \leq l \leq k$.

If $\epsilon$ is a Catalan sequence then there is no vertex $v \in T_{\sigma}$ such that $v \prec R$.

Lemma 6. For a Catalan sequence $\epsilon$ there exists a unique compatible pairing $\sigma$ with the property that $R \preceq v$ for every vertex $v \in T_{\sigma}$. We call it Catalan pairing.

Proof. In the sequence $\epsilon$ let us replace each element +1 by a left bracket "〈" and let us replace each element -1 by a right bracket " $\rangle$ ". We leave it as an exercise to the Reader to check that the pairing $\sigma$ between corresponding pairs of left and right brackets is the unique pairing with the required property.

\subsection{The main bijection}

The main result of this section is the algorithm MainBijection( $T$ ) (with the auxiliary algorithm SmallBijection $(T)$ ) which provides the bijection announced in Theorem 4 . In the remaining part of the article we will show that this algorithm indeed provides the desired bijection.

Remark 7. At the beginning of each iteration of the loop in MainBijection $T$ is a quotient tree $T_{\sigma}$ for some pairing $\sigma$ which is compatible with $\epsilon_{i}$. In order to check it (formally: by induction) we observe that $l_{i}$ edges from each side of the root in the polygonal graph $G_{\epsilon_{i}}$ are among those which were unglued in line 7 of MainBijection. These are the edges which we remove in 8 of MainBijection. Formally, it corresponds to removal of the first $l_{i}$ and the last $l_{i}$ elements from the sequence $\epsilon_{i}$ and it is easily checked that the result is equal to $\left(-\epsilon_{i-1}\right)$. The change of the orientations of the edges in line 9 means the change of sign of the corresponding sequence $\epsilon$, hence after the iteration of the main loop in MainBijection $T=T_{\sigma}$ is a quotient tree corresponding to $\epsilon_{i-1}$.

Remark 8. The operation of reversing the order $<$ in line 9 of MainBijection means that we do not change the labels assigned to the tree $T$ but we change (by reversing) the way we compare them. It follows that for in line 3 and in the function SmallBijection( $T$ ) we consider the set of labels (which is the set of integer numbers) with its usual order < if $m-i$ is even and with the reverse of its usual order if $m-i$ is odd.

Remark 9. Tree $T$ in the algorithms MainBijection and SmallBijection is always a quotient tree $T_{\sigma}$ for some pairing $\sigma$ which is compatible with some sequence $\epsilon$. Each edge of this tree was created from a pair of the edges of the polygonal graph $G_{\epsilon}$; therefore the operation of ungluing in line 9 of SmallBijection should be understood as ungluing of 


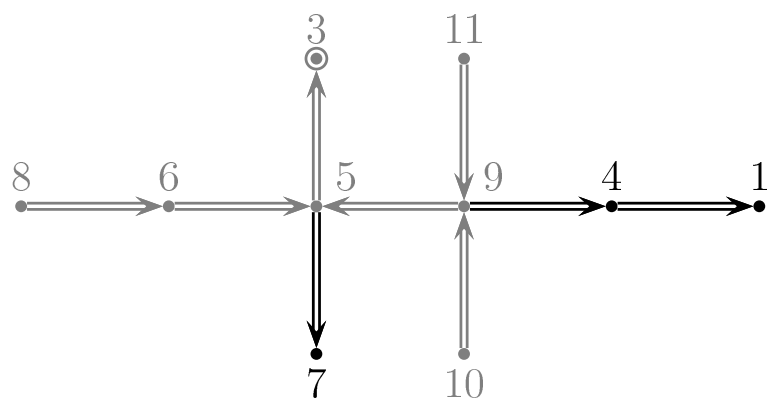

Figure 7: Algorithm MainBijection(T), line 5 Subtree $U$ was marked in gray.

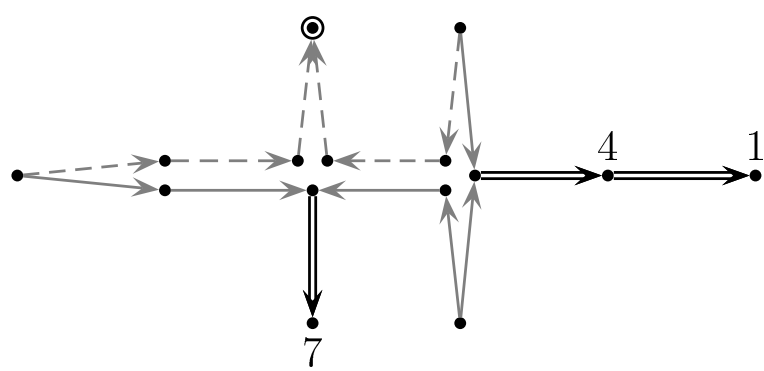

Figure 8: Algorithm MainBijection(T), line 7

these original edges. On a formal level ungluing corresponds to removal of some pairs from the pairing $\sigma$; similarly regluing in line 10 of SmallBijection corresponds to a creation of new pairs in $\sigma$. Similar remarks concern lines 711 of MainBijection.

Remark 10. Lines 16 of SmallBijection compute the bay $B A, C A$ corresponding in the tree $U$ to the leaf $D$.

\section{Proof of the correctness of the small bijection}

\subsection{Statement of the result}

Let $T_{\sigma}$ be a quotient tree and let $<$ be an order on its vertices. We may always label the vertices of $T_{\sigma}$ (for example, with integer numbers) in such a way that the order of the vertices coincides with the order of the corresponding labels. In this way we can view SmallBijection as a map which to a pair $\left(T_{\sigma},<\right)$ (or, more formally, $\left.(\sigma,<)\right)$ associates another pair of this form.

Theorem 11. Let $\epsilon=(\epsilon(1), \ldots, \epsilon(k))$ be a Catalan sequence. The function SmallBijection as described above provides a bijection between

(A) the set of pairs $(\sigma,<)$, where $\sigma$ is a pairing compatible with $\epsilon$ and $<$ is a total order on the vertices of $T_{\sigma}$ compatible with the orientation of the edges; 


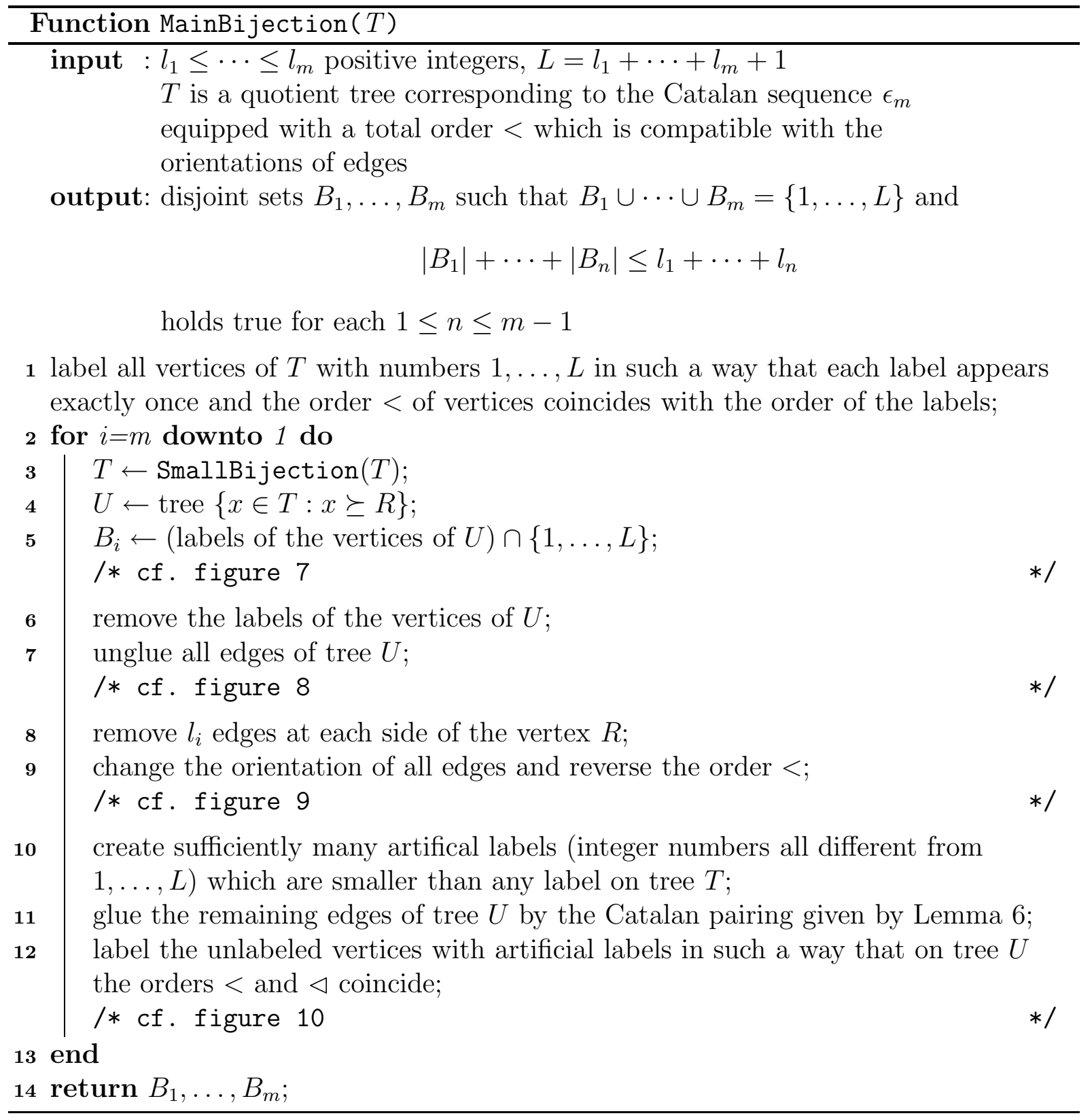




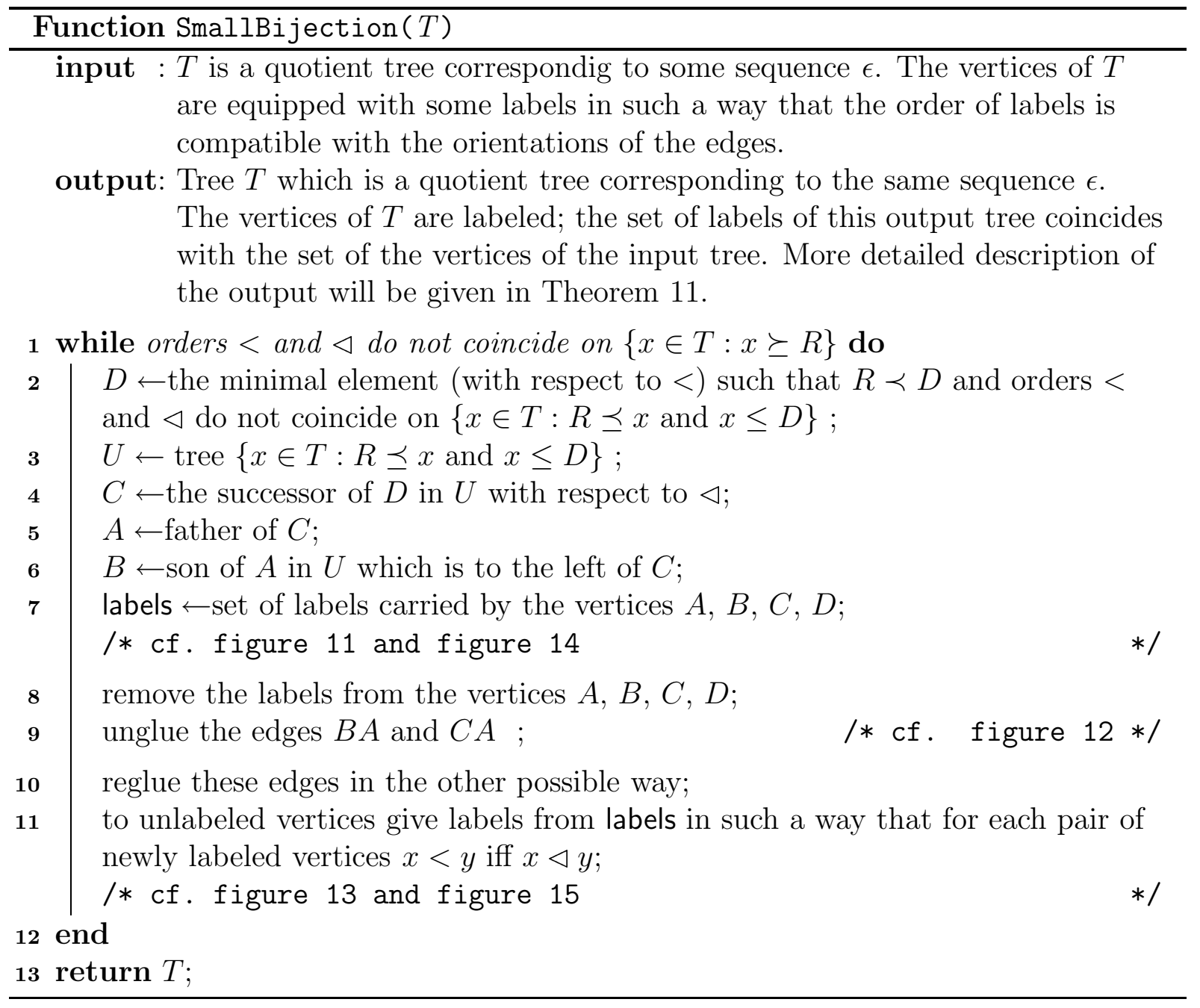




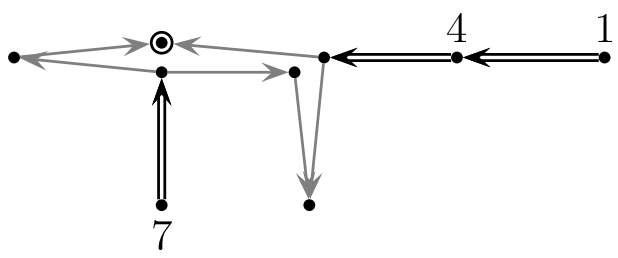

Figure 9: Algorithm MainBijection( $T$ ), line 9 This graph was obtained from Figure 8 by removal of the dashed edges and it can be regarded as a certain polygonal graph $G_{\epsilon^{\prime}}$ with a number of trees attached to it. The orientation of all edges was reversed.

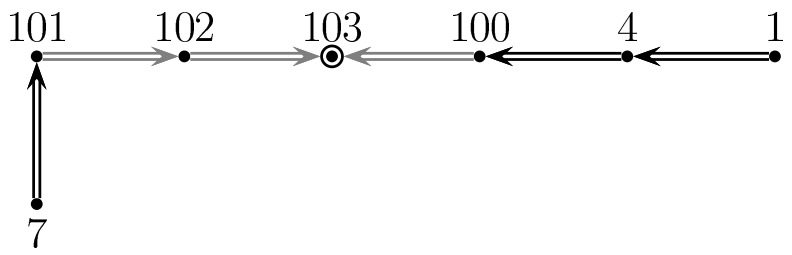

Figure 10: Algorithm MainBijection( $T$ ), line 12. The polygonal graph $G_{\epsilon^{\prime}}$ from figure 9 was glued according to the Catalan pairing. Artificial labels 100-103 were created to label new vertices. The order of the labels was reversed therefore $103<102<101<$ $100<7<4<1$.

(B) the set of pairs $(\sigma,<)$, where $\sigma$ is a pairing compatible with $\epsilon$ and $<$ is a total order on the vertices of $T_{\sigma}$ with the following two properties:

- on the set $\left\{x \in T_{\sigma}: x \succeq R\right\}$ the orders $<$ and $\triangleleft$ coincide;

- for all pairs of vertices $v, w \in T_{\sigma}$ such that $R \npreceq v$ and $R \npreceq w$ we have

$$
v \prec w \Longrightarrow v<w .
$$

The remaining part of this section is devoted to the proof of this theorem.

\subsection{Intermediate triples}

Our strategy is to describe precisely which pairs $(\sigma,<)$ (or alternatively, trees $T$ ) might arise in the intermediate steps of algorithm SmallBijection.

Definition. We call $(\sigma,<, S)$ an intermediate triple if $\sigma$ is a pairing compatible with $\epsilon,<$ is a total order on the vertices of $T_{\sigma}$ and $S$ is one of the vertices of $T_{\sigma}$ with the following properties:

1. $R \preceq S$ and $R \leq S$, where $R$ denotes the root;

2. on the set $\{x: R \preceq x$ and $x \leq S\}$ the orders $<$ and $\triangleleft$ coincide;

3. for all pairs of adjacent vertices $v, w \in T_{\sigma}$ such that $v \prec w$ and $v>w$ we have $R \npreceq v$ and $R \prec w$ and the set $\left\{x \in T_{\sigma}: R \preceq x\right.$ and $\left.S<x<v\right\}$ is empty. 


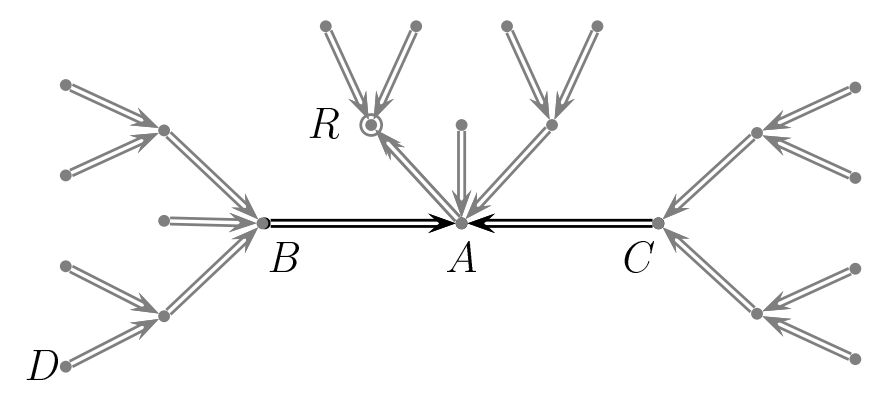

Figure 11: Algorithm SmallBijection( $T$ ), case $D \neq B$. The order of the vertices is given by $R \leq A<B<C<D$. Note that only edges belonging to the subtree $U$ are displayed.

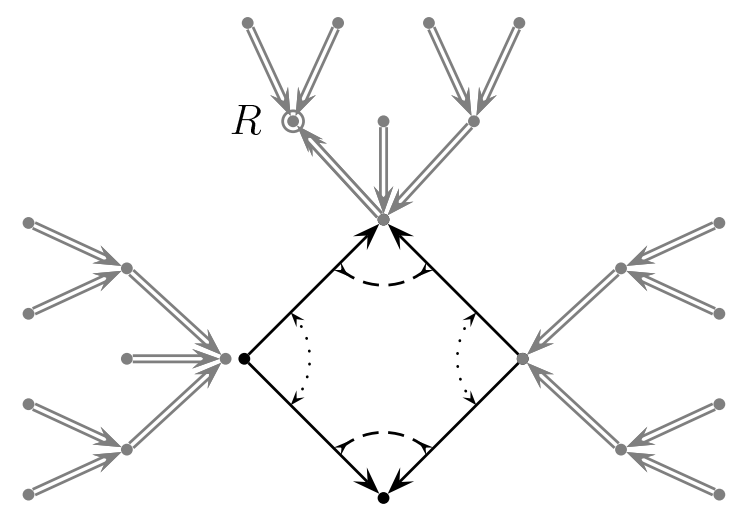

Figure 12: The tree from Figure 11 after ungluing the edges $B A$ and $C A$.

\subsection{Startpoints and endpoints}

Lemma 12. Intermediate triples $(\sigma,<, S)$ for which $S=R$ are in a one-to-one correspondence with the pairs $(\sigma,<)$ which contribute to the set $(A)$ and thus to the possible input data of algorithm SmallBijection.

Proof. Suppose that $(\sigma,<)$ contributes to $(\mathrm{A})$; we set $S=R$. In order to show property (2) of intermediate triples it is enough to observe that if $x$ fulfills $R \preceq x$ then also $R \leq x$, therefore the set $\left\{x \in T_{\sigma}: R \preceq x\right.$ and $\left.x \leq R\right\}$ consists of only one element $R$. The other two properties of intermediate triples hold true trivially.

Suppose that $(\sigma,<, R)$ is an intermediate triple and suppose that there exists a pair of vertices $v, w$ such that $v \prec w$ and $v>w$. With no loss of generality we may assume that the vertices $v$ and $w$ are adjacent (if this is not the case we may find a pair of adjacent vertices $v^{\prime}, w^{\prime}$ such that $v^{\prime} \prec w^{\prime}$ and $v^{\prime}>w^{\prime}$ on the path from the vertex $v$ to the vertex $w)$. In the case $w \leq R$ property (3) shows that $R \prec w$ and property (2) shows that since $R \triangleleft w$ therefore $R<w$ which contradicts $w \leq R$. In the case $w>R$ the set $\left\{x \in T_{\sigma}: R \preceq x\right.$ and $\left.R<x<v\right\}$ contains $w$ which contradicts property (3). In this way we proved that the total order $<$ is compatible with the orientations of the edges. 


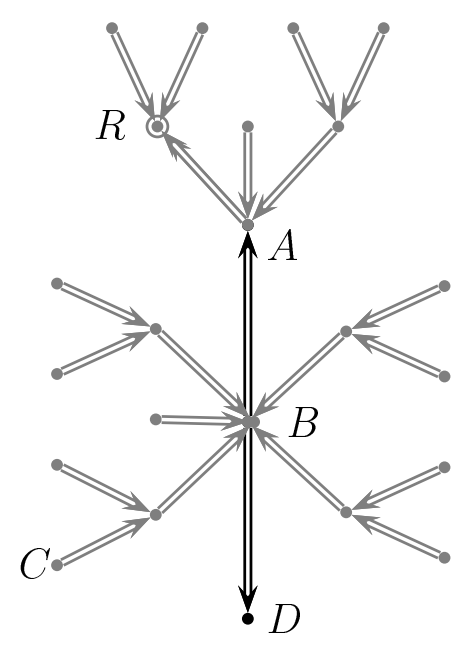

Figure 13: The tree from Figure 11] after regluing the edges $B A$ and $C A$ in a different way. Please notice the change of the labels of the vertices $A, B, C, D$.

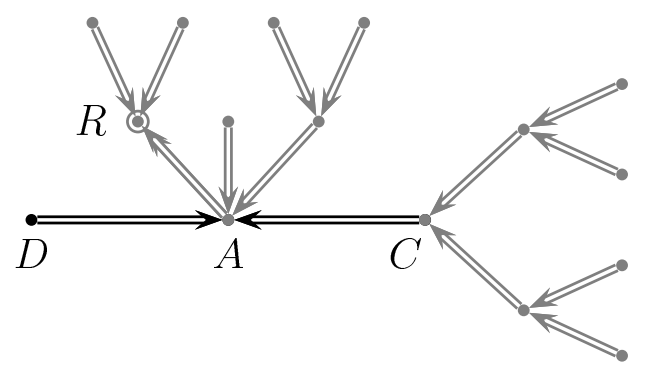

Figure 14: Algorithm SmallBijection( $T$ ), case $D=B$. The order of vertices is given by $R \leq A<C<D$.

Lemma 13. Intermediate triples $(\sigma,<, S)$ for which $S$ is the maximal element (with respect to the order $<$ ) of the set $\left\{x \in T_{\sigma}: x \succeq R\right\}$ are in a one-to-one correspondence with the pairs $(\sigma,<)$ which contribute to the set $(B)$. For such values of $T=T_{\sigma}$ algorithm SmallBijection terminates.

Proof. Suppose that $(\sigma,<)$ contributes to the set $(\mathrm{B})$; we define $S$ to be the maximal element (with respect to $<$ ) of the set $\left\{x \in T_{\sigma}: x \succeq R\right\}$. In order to prove that $(\sigma,<, S)$ is an intermediate triple it is enough to show property (3) of intermediate triples. Suppose that there exist adjacent vertices $v, w$, such that $v \prec w$ and $v>w$; we shall consider now three cases. The first case, $R \npreceq v, w$ is not possible, since then $v<w$ would contradict $v>w$. The second case, $R \preceq v \prec w$ would imply $v \triangleleft w$ and hence $v<w$ again contradicts $v>w$. Therefore, the only remaining possibility is $R \preceq w$ and $R \npreceq v$. It is not possible that $R=w$ since then $v \prec R$ would contradict the assumption that $\epsilon$ is a Catalan sequence. In this way we proved that $R \prec w, R \npreceq v$ which finishes the proof.

Suppose that $(\sigma,<, S)$ is as in the statement of the lemma. In order to prove that $(\sigma,<)$ contributes to (B) it is enough to prove that for all vertices $v, w$ such that $R \npreceq v$ and $R \npreceq w$ we have $v \prec w \Longrightarrow v<w$. If this is not the case then there exist vertices 


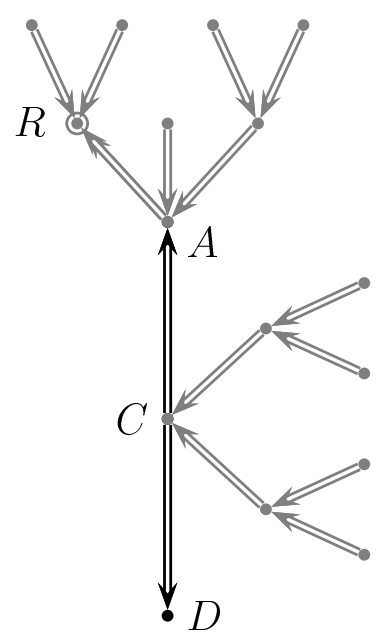

Figure 15: The tree from Figure 14 after regluing the edges $D A$ and $C A$ in a different way. Please notice the change of the labels of the vertices $A, C, D$.

$v, w$ such that $R \npreceq w$ and $v \prec w, v>w$. With no loss of generality we may assume that the vertices $v, w$ are adjacent hence $R \prec w$ contradicts $R \npreceq w$.

\subsection{The forward transformation}

In this section we shall describe a certain invertible operation on intermediate triples which will turn out to be equivalent to the algorithm SmallBijection. After a sufficient number of iterations every intermediate triple corresponding to some element of (A) gets transformed into an intermediate triple corresponding to some element of (B). In this way the operation described in this section (which coincides with SmallBijection) provides a bijection from $(\mathrm{A})$ to $(\mathrm{B})$.

Let $(\sigma,<, S)$ be an intermediate triple and let $D$ be the smallest element (with respect to the order $<$ ) in the set $\{x: x \succ R$ and $x>S\}$. If no such element $D$ exists, this means that the triple $(\sigma,<, S)$ is as in Lemma 13 and hence can be identified with an element of the set $(\mathrm{B})$; in other words our algorithm finished its work.

If $(\sigma,<, D)$ is an intermediate triple, then we iterate our procedure.

We consider now the opposite case when $(\sigma,<, D)$ is not an intermediate triple. Then $S$ is the maximal element (with respect to $<$ ) such that $R \preceq S$ and such that on the set $\left\{x \in T_{\sigma}: R \preceq x\right.$ and $\left.x \leq S\right\}$ the orders $<$ and $\triangleleft$ coincide. Also, $D$ is as prescribed by line 2 of SmallBijection.

Let us denote by $\left(\sigma^{\prime},<\right)$ the pairing and the order which correspond to the value of $T$ in line 11 of algorithm SmallBijection.

Lemma 14. The triple $\left(\sigma^{\prime},<, S\right)$ given by the above construction is an intermediate triple. The above procedure will stop after a finite number of steps.

Proof. We shall consider only the case when $B \neq D$, since the other case is analogous. Conditions (11) and (21) are very easy to verify. To check condition (31) we need to find 
adjacent pairs of vertices $v, w$ on tree $T_{\sigma^{\prime}}$ for which $v \prec w$ and $v>w$. Since condition (3) is fulfilled for the tree $T_{\sigma}$ it is enough to restrict our attention to such pairs which are new, i.e. which were not present on the tree $T_{\sigma}$. Figure 13 indicates one such pair (namely $v=D, w=B$ and it is easy to check that this pair causes no problems) there might be however some other such pairs which were not shown on Figure 12 because $v \notin U$ or $w \notin U$. It is easy to check that such new pairs must fall into one of the following three categories: $C<v<D, w=C$ (causes no problems); or $v=D, A<w<D$ (impossible, since it would imply that in the tree $T_{\sigma}$ the vertex $A$ is adjacent to the vertex $w$ such that $A<w<D$ but $(\sigma,<, S)$ is an intermediate triple, contradiction); or $B<v<C, w=B$ (causes no problems).

Note that in each step of our operation the cardinality of $\left\{x \in T_{\sigma}: R \preceq x\right.$ and $\left.S \leq x\right\}$ decreases. This shows that our procedure will eventually stop.

\subsection{The backward transformation}

In this section we shall describe the inverse of the transformation from Section 3.4 .

Let $(\sigma,<, S)$ be an intermediate triple and let $S^{\prime}$ be the biggest element (with respect to the order $<$ ) of the set $\{x: x \succeq R$ and $x<S\}$. If no such element exists it means that $(\sigma,<, S)$ is as in Lemma 12 hence our algorithm finished its work. If $\left(\sigma,<, S^{\prime}\right)$ is an intermediate triple, we can iterate our procedure.

We consider now the opposite case when $\left(\sigma,<, S^{\prime}\right)$ is not an intermediate triple. It is possible only when condition (3) of an intermediate triple is not fulfilled, namely there is a pair of adjacent vertices $B, D$ such that $B \succ D$ and $B<D$ and $\left\{x: R \preceq x\right.$ and $S^{\prime}<$ $x<D\}$ is a non-empty set. There may be many pairs $B, D$ with this property; let us select the one for which $D$ takes its maximal value (with respect to $<$ ). Since $(\sigma,<, S)$ is an intermediate triple therefore $\{x: R \preceq x$ and $S<x<D\}$ is empty. It follows that the only element which could possibly belong to $\left\{x: R \preceq x\right.$ and $\left.S^{\prime}<x<D\right\}$ is equal to $S$ and therefore $S<D$. In particular,

$$
\{x: R \preceq x \text { and } S<x<D\}=\emptyset .
$$

Let $U$ denote the subtree of $T_{\sigma}$ which consists of the vertices $\{D\} \cup\{x: R \preceq x$ and $x \leq$ $S\}$. Let us change for a moment the orientation of the edge $D B$, as shown on the righthand side of Figure 16 and the right-hand side of Figure 18. It is easy to see that after this change the tree $U$ has the form as considered in Section 2.2 , i.e. the arrows on all edges are pointing towards the root and it has at least two vertices.

Let us consider the case when a corner formed in the vertex $B$ by some edge on the left and the edge $D B$ on the right is a bay (on the right-hand side of Figure 16 this corner corresponds to the pair of edges $u B, D B$ ). In this case let $C$ denote the leaf corresponding to this bay, as described in Section 2.2. We unglue the edges $B A$ and $B D$ and we reglue them in the other way, as we described in Section 3.4 and thus we obtain a tree $T_{\sigma^{\prime}}$ corresponding to some pairing $\sigma^{\prime}$. Figure 17] describes the way how the vertices of the original tree $T_{\sigma}$ are identified with the vertices of $T_{\sigma^{\prime}}$. We shall prove in the following that $\left(\sigma^{\prime},<, S\right)$ is an intermediate triple. 

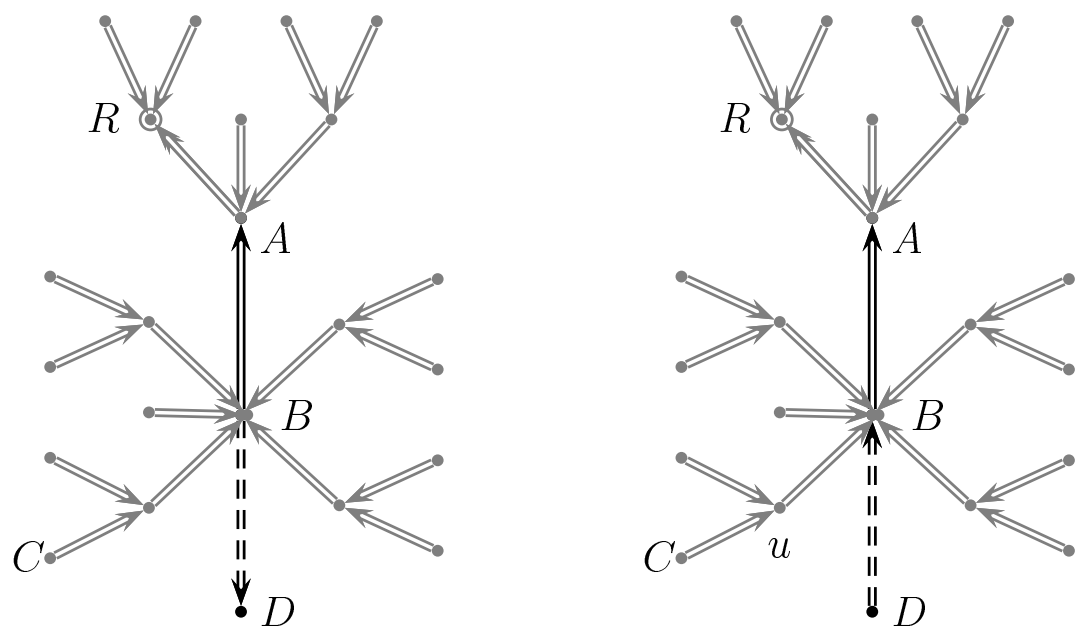

Figure 16: On the left: a tree for which $\left(\sigma,<, S^{\prime}\right)$ is not an intermediate triple. Only vertices belonging to the tree $U$ were shown. On the right: the same tree with the opposite orientation of the edge $D B$. In this case the pair of edges $u B, D B$ forms a bay. The order of the vertices is given by $R \leq A<B<C \leq S<D$.

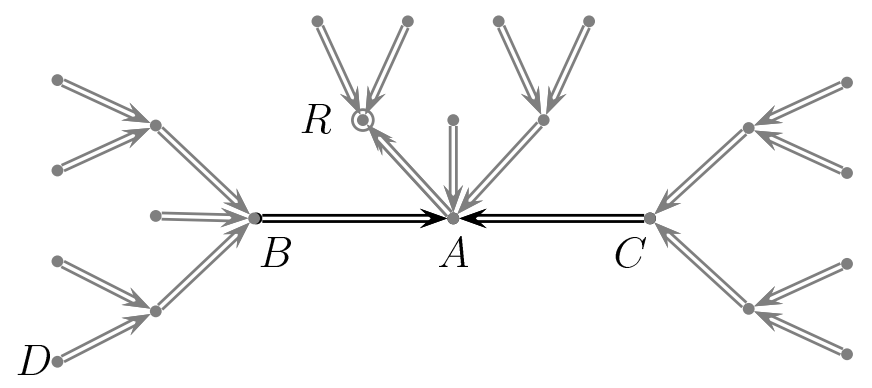

Figure 17: The tree from the left-hand side of Figure 16 after ungluing and regluing differently edges $B A$ and $B D$. Please note that the labels carried by the vertices $A, B, C, D$ have changed.

It remains to consider the case when there is no bay in the vertex $B$ formed by some edge on the left and the edge $D B$ on the right, cf Figure 18. We again unglue and reglue differently the edges $B A$ and $B D$; the resulting tree is denoted by $T_{\sigma^{\prime}}$. The identification of the vertices of $T_{\sigma}$ and $T_{\sigma^{\prime}}$ is presented on Figures 18 and 19, please note that only vertices $A, B, D$ are nontrivially identified.

Lemma 15. The triple $\left(\sigma^{\prime},<, S\right)$ given by the above construction is an intermediate triple. The above procedure will stop after a finite number of steps.

Proof. In the following we consider only the case presented on Figure 16 since the other one is analogous.

Conditions (11) and (2) are very easy to verify. Condition (3) holds true for tree $T_{\sigma}$ hence there are only two reasons why it could fail for the tree $T_{\sigma^{\prime}}$. Firstly, there might be some pair of adjacent vertices $v, w$ on the tree $T_{\sigma^{\prime}}$ for which $v \prec w$ and $v>w$ which 

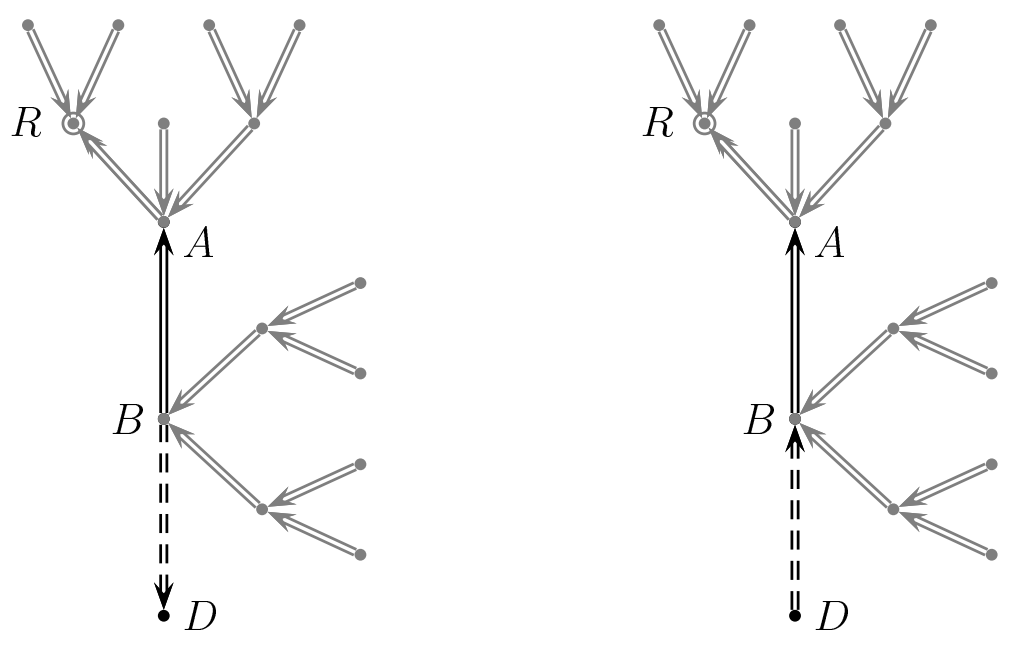

Figure 18: On the left: a tree for which $\left(\sigma,<, T^{\prime}\right)$ is not an intermediate triple. Only vertices belonging to the tree $U$ were shown. On the right: the same tree with the opposite orientation of the edge $B D$. In this case the pair of edges $B A, D B$ does not form a bay. Order of vertices is given by $R \leq A<B<D$.

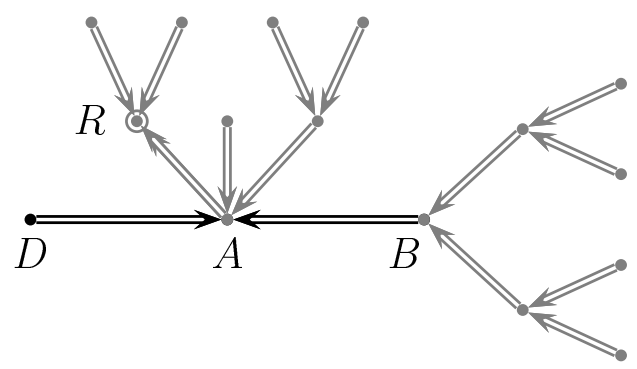

Figure 19: The tree from the left-hand side of Figure 18 after ungluing and regluing differently the edges $B A$ and $B D$. Please note that the labels carried by the vertices $A, B, D$ have changed.

is new, i.e. which is not present in the tree $T_{\sigma}$. There are three possible cases: $v=D$, $C<w<D$ (impossible, since (9) implies $C<w \leq S<D$ and $w \in U$ which contradicts that $C$ is a leaf of the tree $U$ ); or $A<v<D, w=A$ (causes no problems); or $v=C$, $B<w<C$ (causes no problems).

The second reason why condition (3) could fail is that for some pair of adjacent vertices $v, w \notin\{A, B, C, D\}$ such that $v \prec w$ and $v>w$ the set

$$
\{x: R \preceq x \text { and } S<x<v\}
$$

might be non-empty. Since tree $T_{\sigma}$ fulfils condition (3) therefore the only element which could possibly belong to the set $(10)$ is $D$. This, however, is not the case since $D$ was chosen to be the maximal element in the set of the possible values of $v$.

Note that in each step of our operation the cardinality of the set $\left\{x \in T_{\sigma}: R \preceq\right.$ $x$ and $S \leq x\}$ increases. This shows that our procedure will eventually stop. 
The proof of the following lemma is straightforward and we leave it to the Reader.

Lemma 16. The operation described in Section 3.4 and the operation described in Section 3.5 are inverses of each other.

Thus, the proof of Theorem 11 is finished.

We will find the following result useful in Section 4 .

Lemma 17. Let $(\sigma,<, S),\left(\sigma^{\prime},<, S^{\prime}\right)$ be intermediate triples such that $\left(\sigma^{\prime},<, S^{\prime}\right)$ is obtained from $(\sigma,<, S)$ by a number of forward transformations from Section 3.4. We denote $U=\left\{v \in T_{\sigma}: R \preceq v\right.$ and $\left.v \leq S\right\}$ and $U^{\prime}=\left\{v \in T_{\sigma^{\prime}}: R \preceq v\right.$ and $\left.v \leq S^{\prime}\right\}$.

Then $U \subseteq U^{\prime}$. Furthermore, every element of the difference $U^{\prime} \backslash U$ is bigger (both with respect to the order $<$ and $\triangleleft)$ than every element of the set $U$.

Secondly,

$$
\left\{v \in T_{\sigma}: R \preceq v\right\} \supseteq\left\{v \in T_{\sigma^{\prime}}: R \preceq v\right\} .
$$

Inductive proof is straightforward. Please note that, contrary to the order $<$, the order $\triangleleft$ is different on the trees $T_{\sigma}$ and $T_{\sigma^{\prime}}$ and the lemma holds true for both choices of $\triangleleft$.

\section{Proof of the correctness of the main bijection}

We will prove Theorem 4, namely that MainBijection indeed provides the desired bijection.

\subsection{Intermediate points}

Our strategy is to describe possible intermediate outcomes of the algorithm MainBijection.

Definition. We call $\left(T_{\sigma},\left(B_{i+1}, \ldots, B_{m}\right)\right)$ an intermediate point for $i(0 \leq i \leq m)$ if

1. $\sigma$ is a pairing compatible with $\epsilon_{i}$, as defined in (8);

2. $T_{\sigma}$ is a quotient tree with some of the vertices labeled with different elements of $\{1, \ldots, L\}$

3. let $V$ be the set of unlabeled vertices of $T_{\sigma}$; if $i=m$ then $V=\emptyset$, if $i<m$ then $V$ is a tree such that $R \in V, V \subseteq\left\{x \in T_{\sigma}: x \succeq R\right\}$, furthermore for all pairs such that $x \prec y$ and $y \in V$ we also have $x \in V$;

4. for all pairs of labeled vertices such that $x \prec y$ their labels fulfill $x<y$;

5. sets $B_{i+1}, \ldots, B_{m}$ and the set of labels are disjoint and their union is equal to $\{1, \ldots, L\}$

6. $\left|B_{n}\right|+\cdots+\left|B_{m}\right| \geq l_{n}+\cdots+l_{m}+1$ for all $i+1 \leq n \leq m$. 


\subsection{Startpoints and endpoints}

Lemma 18. There is a bijection between the elements of the set ( $\alpha$ ) (thus input data of algorithm MainBijection) and the intermediate points corresponding to $i=m$.

There is a bijection between the elements of the set $(\beta)$ and the intermediate points corresponding to $i=0$ (for which algorithm MainBijection terminates).

\subsection{The forward transformation}

Lemma 19. After each iteration of the loop in MainBijection tuple $\left(\tilde{T},\left(B_{i}, \ldots, B_{m}\right)\right)$ forms an intermediate point, where $\tilde{T}$ denotes the tree $T$ with all artificial labels removed. Futhermore, on the set of the vertices with artificial labels the order $\triangleleft$ coincides with the order of the labels $<$.

Proof. We are going to use backward induction with respect to $i$.

Firstly, observe that in line 3 of MainBijection the computation of SmallBijection is performed on a tree $T$ which is as prescribed in point (A) of Theorem 11 therefore afterwards $T$ is as presecribed in point (B) of Theorem 11 .

Furthermore, Lemma 17] shows that in line 6 of MainBijection all artificial labels will be removed. Therefore all artificial labels in $T$ (equivalently, unlabeled vertices $\tilde{T}$ ) after the iteration of the loop must have been created in line 12.

This shows points (3) and (4) in the definition of an intermediate point.

\subsection{The backward transform}

We shall prescribe now the inverse of the transform prescribed in Section 4.3 (i.e. a single interation of the loop in MainBijection). Since we simply have to reverse all steps of the forward transformation, our description will be quite brief and we shall concentrate only on the most critical points.

As we pointed out in the proof of Lemma 19 all artificial labels in $T$ (equivalently, unlabeled vertices $\tilde{T}$ ) after the iteration of the loop must have been created in line 12, in other words, tree $U$ consists of vertices carrying artificial labels.

Therefore, in order to undo line 12 of MainBijection we simply remove all artificial labels and in order to undo line 11 we unglue all edges with both unlabeled ends.

In order to undo line 9 we change the orientation of all edges and reverse the order of $<$. In order to undo line 8 to both sides of the root we attach $l_{i}$ new edges with appropriate orientations.

In order to undo line 7 we glue the unpaired edges according to the Catalan pairing.

Let $U$ denote the set of unlabeled vertices. In order to undo line 6 we create $|U|-\left|B_{i}\right|$ artificial labels (which are integer numbers different from $1, \ldots, L$ ) which are smaller than any element of the set $\{1, \ldots, L\}$ and we label the elements of $U$ with these artificial labels and the labels from the set $B_{i}$ in such a way that the order $\triangleleft$ of the vertices of $U$ coincides with the order $<$ on the labels. 
In this way tree $T$ is as prescribed in point (B) of Theorem 11 hence undoing line 3 of MainBijection is possible.

Lemma 20. If the above procedure was started for an intermediate point $\left(\tilde{T}^{\text {initial }},\left(B_{i}, \ldots\right.\right.$, $\left.B_{m}\right)$ ) for $i^{\prime}=i-1$ then the resulting tuple $\left(\tilde{T}^{\text {final }},\left(B_{i+1}, \ldots, B_{m}\right)\right)$ is an intermediate point for $i$, where $\tilde{T}$ is the tree $T$ given by the above procedure with all artificial labels removed.

Proof. Only condition (3) is less trivial and needs to be proved. The inclusion (11) shows that after undoing of line 3 every unlabeled vertex $x$ fulfills $x \succeq R$. Furthermore, in tree $T$ the order of the labels is compatible with the orientations of the edges; therefore if $x \prec y$ and $y$ is unlabeled then $x<y$ and $x$ is also unlabeled.

We leave the following lemma as a simple exercise to the Reader.

Lemma 21. Operations described in Sections 4.3 and 4.4 are inverses to each other.

Thus, the proof of Theorem 4 is finished.

\section{Acknowledgments}

I thank Kenneth Dykema for introducing me into the subject. Research supported by the MNiSW research grant P03A 013 30, by the EU Research Training Network "QPApplications", contract HPRN-CT-2002-00279 and by the EC Marie Curie Host Fellowship for the Transfer of Knowledge "Harmonic Analysis, Nonlinear Analysis and Probability", contract MTKD-CT-2004-013389. The author is a holder of a scholarship of European Post-Doctoral Institute for Mathematical Sciences.

\section{References}

[Aag04] Lars Aagaard. The non-microstates free entropy dimension of DT-operators. J. Funct. Anal., 213(1):176-205, 2004.

[AH04] Lars Aagaard and Uffe Haagerup. Moment formulas for the quasi-nilpotent DT-operator. Internat. J. Math., 15(6):581-628, 2004.

[Bro86] L. G. Brown. Lidskiù's theorem in the type II case. In Geometric methods in operator algebras (Kyoto, 1983), pages 1-35. Longman Sci. Tech., Harlow, 1986.

[DH01] Kenneth Dykema and Uffe Haagerup. Invariant subspaces of Voiculescu's circular operator. Geom. Funct. Anal., 11(1):693-741, 2001.

[DH04a] Ken Dykema and Uffe Haagerup. DT-operators and decomposability of Voiculescu's circular operator. Amer. J. Math., 126(1):121-189, 2004.

[DH04b] Ken Dykema and Uffe Haagerup. Invariant subspaces of the quasinilpotent DT-operator. J. Funct. Anal., 209(2):332-366, 2004.

[DY03] Kenneth Dykema and Catherine Yan. Generating functions for moments of the quasi-nilpotent DT operator. Adv. in Appl. Math., 30(3):545-561, 2003. 
[Haa01] Uffe Haagerup. Spectral decomposition of all operators in a $I I_{1}$ factor, which is embedable in $R^{\omega}$. preprint, 2001.

[Haa02] U. Haagerup. Random matrices, free probability and the invariant subspace problem relative to a von Neumann algebra. In Proceedings of the International Congress of Mathematicians, Vol. I (Beijing, 2002), pages 273-290, Beijing, 2002. Higher Ed. Press.

[HP00] Fumio Hiai and Dénes Petz. The semicircle law, free random variables and entropy. American Mathematical Society, Providence, RI, 2000.

[JŚ06a] Artur Jeż and Piotr Śniady. Generalized cauchy identities, trees and multidimensional Brownian motions. Part II: Combinatorial differential calculus. In preparation, 2006.

[JŚ06b] Artur Jeż and Piotr Śniady. Generalized cauchy identities, trees and multidimensional Brownian motions. Part III: Multidimensional arc-sine laws. In preparation, 2006.

[Kre72] G. Kreweras. Sur les partitions non croisées d'un cycle. Discrete Math., 1(4):333-350, 1972.

[Pit75] J. W. Pitman. One-dimensional Brownian motion and the three-dimensional Bessel process. Advances in Appl. Probability, 7(3):511-526, 1975.

[Ran60] George N. Raney. Functional composition patterns and power series reversion. Trans. Amer. Math. Soc., 94:441-451, 1960.

[Śni03] Piotr Śniady. Multinomial identities arising from the free probability theory. $J$. Combin. Theory Ser. A, 101(1):1-19, 2003.

[Spe98] Roland Speicher. Combinatorial theory of the free product with amalgamation and operator-valued free probability theory. Mem. Amer. Math. Soc., 132(627):x+88, 1998.

[ŚS01] Piotr Śniady and Roland Speicher. Continuous family of invariant subspaces for R-diagonal operators. Invent. Math., 146(2):329-363, 2001.

[Sta99] Richard P. Stanley. Enumerative combinatorics. Vol. 2, volume 62 of Cambridge Studies in Advanced Mathematics. Cambridge University Press, Cambridge, 1999.

[VDN92] D. V. Voiculescu, K. J. Dykema, and A. Nica. Free random variables. American Mathematical Society, Providence, RI, 1992.

[Voi96] D. Voiculescu. The analogues of entropy and of Fisher's information measure in free probability theory. III. The absence of Cartan subalgebras. Geom. Funct. Anal., 6(1):172-199, 1996. 